Reprod. Immunol. Biol. 20(1): 33-56.

\title{
Abstracts of the 19th Annual Meeting of Japan Society for Immunology of Reproduction
}

\author{
Held on October 11-12, 2004 \\ at Hotel de Yama, Hakone, Kanagawa \\ (St. Marianna University School of Medicine)
}

\section{Symposium I}

1. Dynamic changes of matrix metalloproteinase and tissue inhibitor of metalloproteinase concentrations in early stage of pregnancy

Hiroaki Shibahara

Jichi Medical School

Objective: Matrix metalloproteinase (MMP) and tissue inhibitor of metalloproteinase (TIMP) play an important role in tissue remodeling systems such as embryo implantation, tumor invasion and tumor metastasis. It would be beneficial if serum concentrations of MMP and TIMP could predict successful implantation following IVF-ET. This study was performed to compare serum MMP and TIMP concentrations between patients with and without the establishment of pregnancy following IVF-ET.

Methods: Ten patients conceived and 10 patients not conceived after IVF-ET were entered. Multiple pregnancies were excluded. Blood samples were obtained 7,14 and 21 days after oocyte retrieval. Serum concentrations of MMP-1, MMP-2, MMP-3, and TIMP-1 were measured using ELISA. These variables were compared with serum E2 and P4 levels. Clinical pregnancies were diagnosed only when fetal heart beat was visualized on ultrasound.

Results: There were no significant differences of patient's age, number of oocytes retrieved, and number of embryos transferred between pregnant and non-pregnant women. TIMP-1 concentrations on day 14 and day 21 in the pregnant group were significantly higher than those in the non-pregnant group [Day14: 223.1 $11.9 \mathrm{vs}$ $177.5 \pm 20.6 \mathrm{ng} / \mathrm{ml}(\mathrm{P}=0.004)$; Day 21: $215.4 \pm 27.8$ vs $181.5 \pm 27.4 \mathrm{ng} / \mathrm{ml}(\mathrm{P}=0.03)]$. TIMP-1 level was also correlated with E2 and $\mathrm{P} 4$ concentrations $(\mathrm{P}<0.0001)$. However, there were no significant differences of MMPs concentrations between the 2 groups. There was no correlation of TIMP-1 level and serum concentration of $\beta$ hCG.

Conclusions: It was demonstrated that patients successfully conceived after IVF-ET showed significantly higher serum concentrations of TIMP-1, which inhibits the activated MMPs, indicating that serum TIMP-1 might be used as one of the predictors of pregnancy in patients treated by IVF-ET.

2. Efficacy of paternal lymphocyte immunization for the treatment of implantation failure in IVF/ICSI treatment

Yoshiharu Morimoto

The Centre for Reproductive Medicine and Infertility, IVF NAMBA CLINIC

Over a decade is going to pass since the invention of in vitro fertilization technique. In our country the birth from ART (artificial reproductive technology) treatment occupies over $1.5 \%$ of all baby born. Since then effort for improvement has been accumulated in drugs. instruments and technology in this field.

However the implantation has been recognized as "a black box" . as the mechanism is too complex with several processes to be clarified. In artificial reproductive medicine, implantation failure remained as a problem. 
In cases of implantation failure, we have checked anti-nuclear antibody, anti DNA antibody, anti-caldiolipin antibody, anti-phosphatidylethanolamine antibody as auto immune disorder mixed lymphocyte test in allogenic immune disorder and platelet agglutination competence test and agglutination tests for disorder of haemostatic system. We have tried treatments such as administration of glucocorticoid for high autoantibody cases, low dose aspirin for haemostatic disorder and paternal lymphocyte immunization for allogenic immune incompatibility.

Over 22 percent is the normal range in mixed lymphocyte test, and the paternal lymphocyte immunization is indicated for the patients blow the range. Immunization is done every two weeks for four times in general .

For the study to investigate efficacy of paternal lymphocyte immunization for the treatment of implantation failure, we selected patients who produced good quality embryos but resulted recurrent implantation failures. The patients with endocrine disorder, uterine abnormality and cases of autoimmune disorder were excluded from the investigation. As a result, the patients who was diagnosed as allogenic immune incompatibility and took immunotherapy conceived as much as the patient without any immune disorder.

Validity of paternal lymphocyte immunization for the recurrent pregnancy loss is still controversial. Furthermore that for implantation failure in ART has not often been studied and more discussion be needed, but this treatment is attractive for the improvement of ART success and further study is anticipated.

\section{The distribution of blood coagulation factor XIII and macrophages in the mid-secretory endometrium and in early placentation decidua}

Toshihiko Asahina, Kayoko Takata, Kinya Takeuchi, Naohiro Kanayama

Hamamatsu University School of Medicine

Macrophage $(\mathrm{m} \phi)$ secretes various physiological active substances. Many $\mathrm{m} \phi \mathrm{s}$ are detected at the human uterus. They have both the local immunosuppressive action beneficial to pregnancy, and the harmful action to extravillous cytotrophoblasts (EVCTs). Recent findings have indicated that serum concentration of macrophage migration inhibition factor (MIF) and macrophage inhibitory cytokine 1 (MIC-1) are decreased in miscarriages. Nevertheless, precise $\mathrm{m} \phi$ distribution at the uterus is unknown. On the other hand, $\mathrm{m} \phi$ secretes subunit $\mathrm{A}$ of blood coagulation factor XIII (XIIIA). XIIIA plays an important role in maintaining pregnancy, and pregnancies of congenital factor XIII deficiency (XIII-deficiency) result in abortion after 5 weeks' gestation without substitute therapy. Now we investigated the distribution of uterine $\mathrm{m} \phi \mathrm{s}$ and XIIIA, as factors that contribute to implantation and/or early placentation.

Methods: (1) Immunohistochemical staining of XIIIA and $\mathrm{m} \phi$ was performed in the human normal non-pregnant endometrium, myometrium, and endometrial cell cultured in serum-free medium. The Welch' $s$ statistical test was used to evaluate the result of populations. (2) Immunohistochemical staining of XIIIA, cytokeratin. fibronectin and so forth was performed in the intrauterine/tubal implantation tissues of normal women and XIII-deficiency. All specimens were obtained with informed consent.

Results: (1) Divided endometrial $\mathrm{m} \phi \mathrm{s}$, secreting XIIIA, were detected in the cultured cell. In the mid-secretory phase, the number of endometrial $\mathrm{m} \phi \mathrm{s}$ was significantly higher than that in the proliferative phase, and $\mathrm{m} \phi \mathrm{s}$ sometimes aggregated within the sub-epithelial region. Although the number of myometrial $\mathrm{m}$ $\phi \mathrm{s}$ was significantly higher than that of endometrial $\mathrm{m} \phi \mathrm{s}$ in the proliferative phase, the latter was significantly higher than the former in the mid-secretory phase. (2) The number of $\mathrm{m} \phi \mathrm{s}$ in the implantation decidua was significantly higher than that in the mid-secretory endometrium. XIIIA-positive $\mathrm{m} \phi \mathrm{s}$ could not be detected in the fibronectin-positive region where interstitial EVCTs were infiltrating. Extracellular XIIIA of normal women was detected at the well-formed cytotrophoblastic shell adjacent to the Nitabuch's layer, while in the specimen of XIII-deficiency, the cytotrophoblastic shell was poorly formed and XIIIA was not detected there.

Conclusion: (1) Human uterine $\mathrm{m} \phi \mathrm{s}$ are numerously present in the myometrium before ovulation. They may migrate to the endometrium and proliferate there after ovulation. The number increases more in the 
implantation decidua, however, the $\mathrm{m} \phi \mathrm{s}$ cannot approach interstitial EVCTs, perhaps by the action of MIF and/or MIC-1. (2) Extracellular XIIIA promotes the early utero-placental adhesion after 5 weeks' gestation perhaps by cross-linking fibrin and fibronectin. Before then, XIIIA is not essential to pregnancy, but it contributes to EVCT migration and proliferation when the cytotrophoblastic shell forms.

\section{Interaction between mother and embryo}

Hiroshi Fujiwara

Department of Gynecology and Obstetrics, Faculty of Medicine, Kyoto University

The attachment of embryo on the endometrial surface and subsequent invasion into endometrial stromal tissues are essential processes for human embryo implantation. The maternal endometrium is considered to receive embryo implantation during the limited period. so-called implantation window. This period may be regulated mainly by ovarian sex steroid hormones such as estrogen and progesterone. However, the precise mechanism for regulation of endometrial receptivity for human embryo remains unclear and there has been few direct evidence to show the periodic changes of human endometrial receptivity during the menstrual cycle.

The attachment assay using human endometrial epithelial cells (EECs) and BeWo cell spheroids, which is a human choriocarcinoma-derived cell line, the majority of spheroids attached on the EECs obtained from women in the mid-secretory phase. However, few spheroids could attach on the EECs derived from other phases, suggesting that some adhesion-interfering substances exit in these EECs. EECs treated with proteases significantly enhanced their ability to receive spheroid attachment, supporting the above speculation. In addition, attached spheroids were significantly increased on the EECs that were co-cultured with PBMC. These findings suggest that immune system as well as proteases secreted from endometrial glands or developing embryo play some roles in regulation of endometrial receptivity for human embryo.

In Matrigel invasion assay, human PBMC promoted murine embryo spreading and invasion. This effect is higher in PBMC derived from pregnant women than in those from non-pregnant women. BeWo cell invasion was also promoted by PBMC derived from pregnant women and chemoattractive soluble factors secreted from PBMC were shown to induce BeWo cell invasion. These invasion-promoting effects were enhanced by HCG treatment. In addition. HCG increased IL-8 production by PBMC, and not LH/HCG receptor, but glycosilated-sites of HCG were speculated to mediate this reaction. Furthermore, human extravillous trophoblasts isolated from primary villous explant culture expressed a chemokine receptor, CCR1. and their invasion was enhanced by RANTES.

These findings suggest that immune system also regulates embryo attachment on EECs and invasion into endometrial stromal tissues in cooperation with endocrine system.

\section{Symposium II}

\section{The gene related gamesomeness}

Naoki Takeshita

Ist Department Obstetrics and Gynecology; TOHO University School of Medicine

Owing to recent advances in molecular biology. Y chromosome genes associated with spermatogenesis have been identified. In 1976. Tiepolo and Zuffardi first reported a correlation between long arm of $Y$ chromosome deletions and azoospermic men. Therefore, several studies demonstrated that deletions in a specific region on the long arm of the $\mathrm{Y}$ chromosome (Yq) result in spermatogenic failure. This region was later identified to be AZoospermic Factor (AZF). At present four different AZF regions (AZFa, AZFb. AZFd and $A Z F c)$ have been reported. In addition, numerous candidate genes of AZF have also been reported by many authors such as DAZ. RBMY. USP9Y, and DBY. Especially DAZ (Deleted AZoospermia), in which the AZFc gene is located, is strongly suspected to be an azoospermic factor. The microdeletion of DAZ on infertile men is 
higher than that in other genes. The human DAZ gene family contains a cluster of DAZ genes and a single autosomal homologue, DAZLA, in which located chromosome 3p24. The DAZL gene is highly homologous to the DAZ gene, with an around $80 \%$ similarity in the coding region of the cDNA. However, the mechanism of DAZLA remains to be elucidated. DAZLA may play an important role in the mitosis and meiosis on human spermatogenesis. Also it is recognized on ovarian tissue. The purpose of this study is to assess the DAZLA gene expression during gametogenesis. Mitochondrial DNA also is considered to related gametogenesis. In this study, we assess to analyze for Y chromosome microdeletion, DAZLA gene, and mtDNA (Mitochondrial DNA). It's so important to have counseling for infertility couple.

\section{Application of vitrification for clinical practice}

Hidekazu Saito

National Center for Child Health and Development

Techniques for in vitro fertilization and embryo transfer have improved a great deal during the last years and as a result, many more pregnancies have been established. This improvement comes accompanied by a higher rate of multiple pregnancy. Multiple pregnancy is associated with various complications including threatened abortion and premature delivery, fetal malformation. need of cervical ligation and caesarean section, greater uterine bleeding at birth. and babies with low birth weight. Decreasing the number of embryos transferred into the uterus is one of the best approaches to prevent multiple pregnancies. As a result. several embryos are left to be stored for future embryo transfer.

Since Whittingham first successful mouse embryo freezing and thawing. many researchers have investigated new ways to improve the method. Various possibilities have been studied like changing the cryoprotectant, or using different freezing and thawing methods. According to the freezing method proposed by Whittingham, it takes 2 to 3 hours for embryos to be frozen with minimal ice formation in embryo blastomeres. To avoid the lethal effect of ice formation. Luyte drew attention to the feasibility of using vitrification for biological material. Rall and Fahy applied the vitrification method to cryopreserve mouse embryos using dimethyl sulfoxide, acetamide, propylene glycol and polyethylene glycol. However. embryos exposed for too long to this cryoprotectant at room temperature were adversely affected by the solution and its high concentration required to properly vitrify embryos. Therefore, this procedure allowed only a very short time for embryos to equilibrate with the cryoprotectant.

Kasai developed a less toxic cryoprotectant, composed of modified phosphate buffer solution, ethylene glycol, ficoll and sucrose. He showed that the optimal equilibration time in the vitrification solution before rapid cooling varied with the ambient temperature according to the criterion of blastocyst formation rate.

Meanwhile, we modified Kasai's cryoprotectant and used trehalose instead of sucrose. We examined the optimal equilibration time when the temperature of cryoprotectant was fixed at $5^{\circ} \mathrm{C}$ aiming to allow longer optimal equilibration time to slowly and steadily handle embryos. To detect possible DNA damage to mouse embryos during our vitrification procedure, we used the sister chromatid exchange technique. This study showed that the lowest DNA damage on embryos occurred when they were equilibrated in the solution for 5 minutes before freezing.

Based on our successful results with vitrification of mouse embryos, we examined the potentiality of applying the method to human embryos. In my lecture today, I would like to explain the key points of the clinical application of vitrification to human embryos and oocytes.

\section{Fertilization failure after ICSI and the effect of ICSI on embryo development}

Kaoru Yanagida

International University of Health and Welfare

Intracytoplasmic sperm injection (ICSI) is effective for functional disorders. such as the disorders of 
capacitation, acrosome reaction. and sperm-egg fusion in the process of fertilization. ICSI is a revolutionary treatment for severe male infertility and unfertilized cases in IVF, since ICSI has the highest fertilization rate. But there are some cases for which ICSI is not effective completely. Here, I discuss about the fertilization failure in ICSI and best plan to fertilization failure, especially artificial oocyte activation. ICSI

1. Fertilization failure after ICSI

Of the 1779 treatment cycles, there have been 100 treatment cycles of completely fertilization failure in ICSI. $13 \%$ of them failed to achieve fertilization after each repeatedly ICSI. It seemed that fertilization failure was due to oocyte activation by analysis with chromatin staining of oocytes. For the failure of oocyte activation, we could apply artificial oocyte activation combined with ICSI. The method of oocyte activation with successful pregnancy reported were $\mathrm{Ca}^{2+}$ ionophore treatment and electrical stimulation. The former was reported by Hoshi et al (1994), the later was reported by Yanagida et al (1999). This procedure was confirmed effectiveness. 8 babies ( 7 pregnancies) were born from the treatment of oocyte activation (electrical stimulation) with ICSI.

2. Effect of ICSI on the embryo development

The incidence of blastocyst formation after ICSI was lower as compared with the IVF group. The process of decondensation of spermatozoa in ICSI was delayed as compared with the IVF. Testicular spermatozoa that contained low SS bonds in nucleoprotein can begin the decondensation as compared with epidydimal and ejacurated spermatozoa. In mice, at 96 hs after insemination or injection, cell number of embryos obtained after ICSI with testicular spermatozoa was high as compared with those obtained with epidydimal spermatozoa. Spermatozoa with poor SS-bonds in nucleoprotein might be favorable to obtain good embryos in ICSI.

\section{Blastocyst culture and development}

Koichi Kyono

Ladies Clinic Kyono

The advantages of blastocysts culture are 1) multiple pregnancy reduction following the decrease of transferred embryos, 2) synchronization of day 5 blastocysts with endometrium, 3) confirmation of embryo quality and development, and 4) decrease of uterine contractions and cervical mucus. Day 5 blastocysts transfer rate has been increased with the advent of newer culture systems. But now, It is not clear which is better in clinical pregnancy rates per oocyte retrieval between on day 3 and day 5 embryos transfer. I would like to state the outcome of the present blastocysts culture and development, and the future strategy of improvement of acquirement of good quality oocytes and blastocysts .and male factor, luteal support.

\section{Influence to the multipe pregnancy of ART}

\section{Minako Matsumoto}

Seirei Hamamatsu General Hospital

With the recent spread of assisted reproductive technology (ART), about $1 \%$ of newborns in Japan are born by ART, and the incidence of multiple pregnancy is increasing. Compared with single pregnancy, multiple pregnancy shows a high incidence of maternal complications and premature deliveries and is an important problem in perinatal medicine. Among multiple pregnancies, super twins (triplets or more) and monochorionic multiple pregnancy have particularly high risks. Super twins have been conventionally managed by limiting the number of transferred embryos. After introduction of blastocyst transfer with high selectivity of good embryos, the incidence of monochorionic multiple pregnancy has been increasing. At our hospital, IVF-ET has been performed since 1989. Initially, the multiple pregnancy rate was $36.8 \%$, and there were 4 quadruplet pregnancies. Reflecting these results, we limited the number of transferred embryos to 3 in 1991 and to 2 in 1998. However, since the introduction of blastocyst transfer in 1999, the incidence of monochorionic multiple pregnancy has been increasing. Therefore. we evaluated the multiple pregnancy rate after limitation of the 
number of transferred embryos at our department and the transfer time and chorion of embryos in multiple pregnancy.

1. In fresh embryo transfer, when the number of blastocysts or morulae was limited to 1 , the pregnancy rate was $31.9 \%(30 / 94)$, and the multiple pregnancy rate was $3.3 \%(1 / 30)$. When the number was limited to 2 , the pregnancy rate was $44 \%(81 / 184)$, and the multiple pregnancy rate was $22 \%(18 / 81)$. Compared with the increase in the pregnancy rate, the increase in the multiple pregnancy rate was marked. In particular, the multiple pregnancy rate after transfer of 2 good embryos or more, the multiple pregnancy rate in females aged $\leq$ 35 years was more than $40 \%$, which was markedly higher than the multiple pregnancy rate in females aged $35-40$ years $(10 \%)$ and that in females aged $\geq 40$ years $(0 \%)$.

2. In blastocyst transfer in fresh embryo transfer, $21 \%(4 / 19)$ of females with multiple pregnancy showed monochorionic multiple pregnancy ( 2 with MD twins and 2 with DT triplets). This incidence was significantly higher than that $(4.2 \%, 3 / 71)$ in split embryo transfer. In cryopreserved/thawed embryo transfer, MD twins were also observed after blastocyst transfer.

When ART is performed, the number of embryos transferred should be minimized. In addition. ART should be performed with informed consent obtained after adequate explanation of the risks and incidence of multiple pregnancy including monochorionic twins.

\section{Poster Session}

1. Selective passage through the uterotubal junction of sperm from a mixed population produced by chimeras of calmegin-knockout and wild-type male mice

Masaru Okabe ${ }^{1}$, Tomoko Nakanishi ${ }^{2}$, Ayako Isontani ${ }^{1}$, Ryo Yamaguchi ${ }^{1}$, Masahito Ikawa ${ }^{1}$, Tadashi Baba ${ }^{2}$. Susan Suarez ${ }^{3}$

${ }^{1}$ Genome Information Research Center; ${ }^{2}$ Tsukuba University; ${ }^{3}$ Cornell University

Loss of calmegin, a testis-specific putative chaperone protein of the endoplasmic reticulum. leads to male sterility because the sperm show defects in migration into the oviduct and do not bind to the zona pellucida. To clarify the mechanism of defective migration, $\mathrm{XY}-\mathrm{XY}$ chimeras were produced by aggregating wild-type embryos with embryos of transgenic mice lacking functional calmegin genes and expressing green fluorescent protein (GFP) in their acrosomes. Chimeric ejaculates contained wild-type non-fluorescent sperm as well as sperm with GFP-tagged acrosomes and the defective calmegin gene. Transgenic, wild-type, and chimeric males were mated to wild-type females; however, only wild-type sperm were ever found within the oviducts. Calmegin knockout sperm, even when they were combined in chimeric ejaculates with wild-type sperm. remained outside of the uterotubal junction (UTJ). These findings indicate that the presence of wild-type sperm cannot compensate for the inability of calmegin knockout sperm to enter the oviduct and that successful ascent into the oviduct depends on the capabilities of individual sperm.

\section{The expression of oocyte activation in spermaids}

Fumi Tanaka, Ai Kazami, Kahei Sato

Depatment of Applied Life Science, Graduate School of Bioresource Sciences, Nihon University, Kanagawa, Japan

Spermatids, precursor of male gametes, are known to possess the potential to achieve fertilization and embryonic development when injected into eggs. However, injection of round spermatids alone seldom activates eggs in the mouse. The unification of sperm and egg leads to distinctive series of cytosolic $\mathrm{Ca}^{-}$oscillations that are prerequisite for normal embryo development. It is reported that elongated spermatids have the potential of oocytes activation. Although activating abilities of spermatids expresses during spermiogenesis, the exact time remains unknown. 
Thus, in this study, we investigated the oocyte activation abilities of each stage of spermatids during spermiogenesis: round, elongating or elongated spermatids were injected into mature oocytes without an artificial activation.

Used oocytes were collected from oviducts of superovulated mature B6D2F1 females. The various stages of spermatids were collected from the testis of mature B6D2F1 males. Round, elongating or elongated spermatids were isolated and injected into M II oocytes without any artificial activation, using the micromanipulator. For the verification that round spermatids has the potency of development, when round spermatids were injected, some oocytes were activated artificially. The activation was performed with $7 \% \mathrm{EtOH}$ for 5 minutes before injection. The microinjection of spermatids carried out in modified HTF containing $4 \mathrm{mg} / \mathrm{ml}$ BSA, using the glass pipette $(\sim 10 \mu \mathrm{m}$ inner diameter $)$. The oocytes injected with spermatids were cultured in $\mathrm{KOSM}$ medium at $37^{\circ} \mathrm{C}$. under $5 \% \mathrm{CO}_{2}$ in air and observed every $24 \mathrm{~h}$.

Seventy-six percent of mouse oocytes microinjected with testicular spermatozoa survived and $69.9 \%$ of these were fertilized. Round spermatids could not activate the oocytes without an artificial activation. On the other hand, the $55.5 \%$ of oocytes injected round spermatids with artificial activation were fertilized. Unlike round spermatids, when elongating spermatids were injected into oocytes, the $12.2 \%$ of oocytes activation was observed. After the injection of elongated spermatids, the $32.9 \%$ of oocytes were activated. After the injection of epididymal spermatozoa into the oocytes as a control, the $76.4 \%$ of oocytes were fertilized. But these spermatids were less efficient than testicular spermatozoa.

\section{3. development of embryo injected with secondary spermatocyte in mice}

Ai Kazami, Naoki Okamoto. Yorino Sato, Fumi Tanaka, Kahei Sato

Nihon University

It was previously reported that the nuclei of round spermatids microinjected into mature oocytes were able to contribute to normal fertilization and followed by embryonic development in mouse. This indicates that the genomic imprinting of male germ cells were completed by the round spermatids, the cells have finished second meiosis and have a haploid nuclei. On the other hand, secondary spermatocytes, which were in second meiosis and a $2 \mathrm{~N}$ haploid, were also conceive to complete genomic imprinting because secondary spermatocyte-injected mature oocytes were capable of fertilization and developing to live fetus. However the abilities of secondary spermatocyte to fertilize and to activate oocyte have been not investigated yet. Therefore this study examined the ability of secondary spermatocytes to initiate fertilization when they' re injected into mature oocytes, in order to investigate the abilities of secondary spermatocytes to fertilize and to activate oocytes.

B6D2F1 female mice (6 weeks) were superovulated by PMSG and hCG and cumulus cell-oocyte complexes were collected from ampullae of them. Oocytes were freed from cumulus cells by $0.1 \%$ hyaluronidase. Spermatogenic cells were aspirated the testis of B6D2F1 mature male mice. Secondary spermatocytes were selected according to the shape and the size of cells. The cells that have $14 \mathrm{~mm}$ cell size and $8 \mathrm{~mm}$ diameter of nucleus were regarded as secondary spermatocytes. Presumptive secondary spermatocytes were microinjected into mature oocytes using micromanipulator. After $1.5 \mathrm{hr}$ from injection, oocytes were activated artificially by soaking into $7 \%$ ethanol for $5 \mathrm{~min}$. Then eggs were cultured for 3-5 hours and counted their pronuclei and polarbodies. Those with polar bodies and pronuclei within the ooplasm were considered fertilized. Those were observed their development. Some eggs were fixed and stained their chromosomes in order to investigate their karyotype.

Two pronuclei and three polarbodies were formed about $20 \%$ of eggs of injected oocytes. It was regarded the eggs with two pronuclei and three polarbodies (one was first polarbody and another one was second polarbody derived from oocyte. and the remainder of them was the pseud-polarbody derived from injected spermatocyte) as normally fertilized zygotes. Some embryos developed morula/blastocyst stage. These results showed that secondary spermatocytes have ability of normal fertilization. 


\section{Analysis of androgenic effect for the expression of transition protein (TP)}

Shinji Komori ${ }^{1}$, Nozomi Kanazawa ${ }^{2}$, Kazuko Sakata ${ }^{1}$, Kentaro Nakanishi ${ }^{1}$, Koji Koyama ${ }^{1}$

${ }^{1}$ Hyogo College of Medicine; ${ }^{2}$ Hyogo College of Medine, Institutefor Advaced Medical Sciences

Aim: Transition protein 1 and 2 (TP1,2) play an important role in condensing the chromosome during spermatogenesis. In the experiment with knockout mice of TP 1 and TP 2, a spermatogenic failure was pointed out, suggesting that the expression of TP1 and TP2 are important for spermatogenesis. On the other hand, androgen (A) is an important factor for development of the testis and plays a crucial role in spermatogenesis. Therefore, the androgenic effects via androgen receptor (AR) for the expression of TP1 andTP2 were analyzed in this study.

Methods: 1) On the basis of a DNA database, the 5 ' region of TP1 and TP2 were searched and isolated by using PCR. 2) The isolated 5' region of TP1 and TP2 were cloned into a reporter plasmid with luciferase gene. Human AR genes with various CAG repeats (12.15, 22 or 43 repeats) were also cloned into expression vectors. These plasmids were cotransfected into COS7 cells by electroporaticn. Luciferase activities were measured with or without dihydrotestosterone (DHT). 3) The gel shift assay of 5' region of TP1 and TP2 were performed for analyzing the binding region.

Results: 1) The 5' region of TP1 gene (1150 bp) and TP2 gene (1250 bp) were successfully isolated. 2) In vitro expression experiments, the increase of luciferase activity was identified in COS 7 cells transfected AR with $22 \mathrm{CAG}$ repeats (wild type). On the other hand, any increase of luciferase activities were not identified in COS 7 cells transfected AR with excessively short CAG repeats of 12 and 15 or with an excessively long CAG repeat of 43.3$)$ The 5 ' region $(-754 \sim-471 \mathrm{bp})$ of TP1 and the 5 ' region $(-1218 \sim-858 \mathrm{bp})$ of TP2 were respectively identified as the binding site of A-AR complex by the gel shift assay.

Conclusion: In this study, we demonstrated first time that both $A$ and the length of CAG repeats of AR affected the expression of TP1and TP2. The specific 5' region of TP1 and TP2 was identified as the binding site of A-AR complex. In summary, androgen seems to affect spermatogenesis via the expression of TPland TP2.

\section{Molecular analysis of androgen receptor in infertile males}

Kentaro Nakanishi, Shinji Komori, Kazuko Sakata. Hiroyuki Kasumi, Koji Koyama Hyogo College of Medicine

Aim: Androgen plays a crucial role in testis development and spermatogenesis. Defects of androgen receptor (AR) may lead to various disorders of sexual development. For instance. male infertility results from certain mutations of the AR gene. Small or large numbers of CAG repeats in the AR gene are associated with various diseases, such as Kennedy disease and prostate cancer. In the present work, we analyzed point mutations and the number of CAG repeats in the AR gene in azoospermic and oligozoospermic men.

Methods: Genomic DNA was isolated from 117 oligozoospermic and 19 azoospermic men. The AR gene was amplified by PCR and sequenced.

Results: We found that 9 of 117 oligozoospermic men had 14 or 15 CAG repeats, whereas 136 normal fertile men had more than 16 CAG repeats. No mutations of the AR gene were identified in either azoospermic or oligozoospermic men analyzed.

Conclusion: Our results showed that there is a linkage between short CAG repeats in the AR gene and the failure of spermatogenesis. Point mutations of the AR gene are rare in infertile males with spermatogenic failure.

\section{Presence of $\mathrm{O}$-linked carbohydrate in the human male reproductive tract $\mathrm{CD52}$}

Akiko Hasegawa ${ }^{1}$, Koichi Ito ${ }^{2}$. Kanako Ukita ${ }^{1}$. Shinji Komori ${ }^{1.2}$, Koji Koyama ${ }^{1.2}$

${ }^{1}$ Laboratory of Developmental Biology and Reproduction, Institute for Advanced Medical Sciences, Hyogo College of Medicine; ' Department of Obstetrics and Gynecology; Hyogo College of Medicine 
CD52 is a kind of glycosylphosphatidylinositol (GPI) anchor protein occurring in lymphocytes and the male reproductive tract (mrt) including sperm and seminal plasma. The molecule is an antigen recognized by a complement-dependent sperm-immobilizing antibody occurring in an infertile patient. It has been shown that mrtCD52 contains a unique N-linked carbohydrate that does not cross-react with other tissues. In this study, we investigate whether $\mathrm{O}$-linked carbohydrate as well as $\mathrm{N}$-linked carbohydrate is present in mrtCD52 using specific lectins and anti-CD52 core peptide antiserum. The lectins, PNA and RCA120, which recognize O-linked carbohydrate, reacted to mrtCD52 and showed a quite similar polymorphic reaction pattern to that by the anti-CD52 peptide antiserum in western blotting analysis on two-dimensional SDS-PAGE. The PNA-reactive spots disappeared after removal of O-linked carbohydrate, but not after removal of $\mathrm{N}$-linked carbohydrate. These results suggest that $\mathrm{O}$-linked carbohydrate is present in mrtCD52. The moiety may possibly contribute to a specific antigenic epitope of mrtCD52.

7. Gender differences in anti-sperm immunity: a comparison of the activity of sperm-immobilizing antibodies between immunologically infertile couples

Hiroaki Shibahara, Yasuko Shiraishi, Kumiko Kikuchi, Yuki Hirano, Tatsuya Suzuki, Satoru Takamizawa, Mitsuaki Suzuki

Jichi Medical School

Objectives: It has been shown that anti-sperm antibodies (ASA) are produced both in males and females. Some of ASA significantly relate with refractory infertility by inhibiting sperm passage through female reproductive tract or by blocking sperm-egg interaction. In some autoimmune diseases, disease incidence and disease severity have been shown to differ between males and females. In the present study, the activity of sperm-immobilizing antibodies (SIA) was compared between infertile males and females.

Materials and methods: Between May 1999, and Dec. 2003. SIA in the sera of 887 infertile females and those on the sperm surface of 1072 infertile males were evaluated. In some cases, the post-coital test (PCT) was performed. Pregnancy rate by the treatments, including timed-intercourse (TI), AIH, IVF-ET, and ICSI-ET, was compared.

Results: The incidences of SIA in females and males were $2.8 \%(25 / 887)$ and $0.9 \%(10 / 1072)$, respectively. There was a significant difference between the 2 groups $(\mathrm{P}<0.005)$. Three $(18.8 \%)$ of 16 females with SIA, none $(0 \%)$ of 5 males with SIA, and $42(76.4 \%)$ of 55 patients without SIA had the normal results in PCT. There was no difference between the former 2 groups $(\mathrm{P}>0.05)$. After the treatments, $13(54.2 \%)$ of 24 females with SIA and $8(80.0 \%)$ of 10 males with SIA conceived $(\mathrm{P}>0.05)$. In total. 15 pregnancies from 13 females and 10 pregnancies from 8 males were established. In females, $2(13.3 \%), 2(13.3 \%), 10(66.7 \%)$, and $1(6.7 \%)$ pregnancies were established by TI, AIH. IVF-ET, and ICSI-ET, respectively. In males, $1(10 \%), 1(10 \%), 2$ $(20 \%)$, and $6(60 \%)$ pregnancies were established by TI, AIH, IVF-ET, and ICSI-ET, respectively. There was a significant difference of the ICSI-ET pregnancy rates between the 2 groups $(\mathrm{P}<0.005)$.

Conclusions: Disease incidence of ASA, especially SIA, seems to differ between males and females. The significantly higher necessity of ICSI-ET indicates that some ASA on the sperm surface cause fertilization failure. These findings suggest that there are gender differences in anti-sperm immunity in human.

\section{Immunogenicity of recombinant hamster ZPA protein in female hamsters}

Masateru Hori ${ }^{1}$, Nozomi Kanazawa ${ }^{2}$, Kanako Ukita ${ }^{2}$. Hideaki Sawai ${ }^{2}$. Koji Koyama ${ }^{1}$

${ }^{1}$ Department of Obstetrics and Gynecology, Hyogo College of Medicine; ${ }^{2}$ Laboratory of Developmental Biology and Reproduction, Institute for Advanced Medical Sciences, Hyogo College of Medicine

Introduction: In 1976, it was reported that hamster zona pellucida contained a promising candidate antigen for developing a contraceptive vaccine. However. cDNAs coding for hamster zona proteins except for ZPC have not been cloned. The objective of this study is to isolate the gene coding for hamster ZPA (hamZPA). produce 
recombinant hamZPA, and examine its immuno-contraceptive efficacy in a homologous system.

Methods: HamZPA cDNA was isolated by a degenerate RT-PCR technique using mRNA prepared from 3-week old hamster ovaries and primers designed based on nucleotide sequence of highly-conserved regions among other species. A partial recombinant hamZPA protein (rec-hamZPA) was produced in Escherichia coli using pET21 vector and used for immunization of female hamsters. After the third injection, sera were collected and assayed for antibody production by western blotting and immunofluorescent staining.

Results: A 747 base-pair partial hamZPA cDNA encoding 249 amino acids was isolated. The homology of hamZPA with ZPA of rat, mouse, human and pig was $69.0,64.7,53.0$ and $44.9 \%$. respectively. The antisera produced by immunization of hamsters with the rec-hamZPA specifically reacted to native hamster zona pellucida.

Conclusion: These results suggested that rec-hamZPA is useful to study a contraceptive vaccine in the homologous system.

\section{Are in vitro fertilized eggs able to secret soluble HLA-G?}

Noriko Sageshima ${ }^{1}$, Hirotsugu Hashimoto ${ }^{2}$. Yamashita Masanori ${ }^{3}$, Takanori Syobu ${ }^{1}$. Motoko Omura ${ }^{1}$, Katsuhiko Hatake $^{1}$, Daniel E Geraghty ${ }^{4}$, Akiko Ishitani ${ }^{1}$

${ }^{1}$ Nara Medical University; ${ }^{2}$ ASKA Lady's Clinic; ${ }^{3}$ Yamashita Lady's Clinic; ${ }^{4}$ Fred Hutchinson Cancer Research Center

HLA-G is thought to be involved in protecting the fetus from the maternal immune cells. Recently, Fuzzi et al reported that in-vitro fertilized eggs secreted soluble HLA-G (sHLA-G), and only the eggs secreting HLA-G were able to be successfully implanted. After this report, Lierop et al attempted to duplicate these results. although they were not able to demonstrate same results. Since this finding could have a significant impact on in vitro fertilization, we decided to investigate this question using novel reagents.

Method: We prepared 106 culture supernatants that were collected after 3 days culture or 4-6 days culture following in vitro fertilization. These samples were measured by sandwich ELISA using the specific monoclonal antibodies for HLA-G, $87 \mathrm{G}$ and MEMG/9.

Results: We could correctly detect soluble HLA-G in control samples that contained above $5 \mathrm{ng} / \mathrm{ml}$ of soluble HLA-G. However, we were unable to detect soluble HLA-G in all of samples we investigated. and to find any relation between actual value and successful implantation.

Discussion: Fuzzi et al did not show a standard curve nor the method of purification of soluble HLA-G protein. Lierop et al reported that they failed to detect HLA-G in any culture supernatant of in vitro fertilizes egg. even though their ELISA method could detect above $1.25 \mathrm{ng} / \mathrm{ml}$ of soluble HLA-G. Our date supports the results of the Lierop study.

\section{The variation of appearance of uterine NK cells in an experimental model of human endometrium}

Rui Matsuura, Takashi Murakami. Yuka Ozawa, Yukihiro Terada, Kunihiro Okamura

Tohoku University Graduate School of Medicine

BACKGROUND: We have made an experimental model of human endometrium by using immunodeficient NOD/SCID/ $\gamma$ cnull (NOG) mice, which lack both $\mathrm{T}$ and B lymphocytes and NK cell activity. It has been said that large granulated lymphocytes increase in human secretory phase endometrial stroma. These lymphocytes are known to have an unusual antigen. CD56-positive CD16-negative NK cells, which have different antigen from those present in the peripheral blood. The variation of appearance of these uterine NK cells were observed by using this model.

METHOD: Proliferative phase endometrium was transplanted into the subcutaneous space of ovariectomised NOG mice. Mice were treated with $17 \mathrm{~b}$-estradiol (E) for the first 14 days after transplantation. followed by E plus progesterone (P) for the next 14 days. The implanted endometrial fragments were extracted at 
the different time points, and immunohistochemical methodology was done to identify the lymphocytes appeared in the transplanted tissues.

RESULTS: Lymphocytes appeared in the transplanted tissues were stained with human antigen. Some human CD56-positive cells were detected in stroma of the tissue before transplantation. However, they had disappeared by 14 days after transplantation. A few were observed at 21 days, then they significantly increased in number in stroma by day 28 .

CONCLUSIONS: It is suggested that CD56-positive uterine NK cells were in situ proliferation in endometrial stroma rather than derived from the peripheral blood.

\section{Endometrial hyperplasia in IL-2 receptor beta-chain transgenic mice}

Ken Kusakabe, Zhong-Lian Li, Yoshinori Otsuki

Osaka Medical College

IL-2 receptor is composed of alpha. beta and gamma-chains and IL-2 receptor beta-chain is essential for the high affinity between IL-2 receptor and IL-2 or IL-15 and for signal transduction. In IL-2 receptor beta-chain transgenic (Tg2Rbeta) mice, cystic changes are frequently observed in uterus. The present study was conducted to examine the pathological changes in the uterus of Tg2Rbeta mouse and the involvement of immunological system in the causes.

Wild type (WT) or Tg2Rbeta mice were determined by genotyping assay of tail tissue. Uterus and blood were collected at young age ( $8-12 \mathrm{wks})$, middle age (24-32 wks) and old age (over $40 \mathrm{wks})$, respectively. By immunohistochemistry, estrogen receptor (ER) alpha, proliferating cell nuclear antigen (PCNA) and IL-2 receptor beta-chain were examined in the uterus, and by ELISA. the serum revel of estradiol was measured. Appearances of natural killer (NK) cells and macrophage were examined by immunohistochemistry and Western blotting, and concentration of tumor necrosis factor (TNF)-alpha in uterus, which is derived from NK cells, was measured by ELISA.

Endometrial hyperplasia (EHP) was frequently observed in $83.3 \%$ of old $\mathrm{Tg} 2 \mathrm{R}$ beta mice, while only $50.0 \%$ in old WT mice, and the incidences of EHP increased depending on age in both types of mice. Cystic endometrium of EHP-caused Tg2Rbeta mice was positive for PCNA. No differences were observed in the serum level of estradiol and expression of ER alpha in uterus. In addition, NK cells and macrophages could not be detected and concentration of TNF-alpha in uterus was same level in both types of mice. On the other hand, endometrium in Tg2Rbeta mice was positive for IL-2 receptor beta-chain obviously. although it was weakly expressed in the endometrium of WT mice.

We demonstrated that there was spontaneous EHP in Tg2Rbeta mice. The onset of EHP in Tg2Rbeta mice was suggested to be estrogen-independent, and to be associated with direct affect of IL-2 or IL-15 though IL-2 receptor beta-chain in endometrium. Tg2Rbeta mouse may be a useful model for the study of EHP.

\section{Histone acetylation is involved in differentiation of human endometrial glandular cells accompanied with induction of an immunosuppressive protein glycodelin}

Hiroshi Uchida, Tetsuo Maruyama, Toru Arase. Masanori Ono, Takashi Nagashima, Hirotaka Masuda. Hironori Asada, Yasunori Yoshimura

Dept. of Obstetrics and Gynecology, Keio University School of Medicine

Glycodelin has an immunosuppressive activity against natural killer cells. Since glycodelin shows a prominent expression in the implantation window of endometrial glandular cells (EGCs), it is though to be a differentiation marker of EGCs. Histone acetylation and deacetylation. which is controlled by histone acetylase and histone deacetylase (HDAC) respectively, plays a fundamental role in gene transcription. To address the role of histone acetylation in the regulation of glycodelin gene transcription and differentiation of human endometrium. we examined the effect of two types of histone deacetylase inhibitors (HDACi), trichostatin A and 


\section{Abstracts of the 19th Annual Meeting}

suberoylanilide hydroxamic acid N-hydroxy-N'-phenyloctanediamide, on the glycodelin expression and differentiation of human endometrial adenocarcinoma cell line (Ishikawa). Expression of glycodelin mRNA and protein was induced by HDACi in a time- and dose-dependent manner in Ishikawa cells. Expression of other endometrial gland differentiation marker protein such as leukemia inhibitory factor and interleukin-1 receptor was also enhanced by HDACi at both mRNA and protein levels. Reporter assay using glycodelin promoter region revealed that the promoter activity was enhanced by HDACi treatment in a dose-dependent manner in a similar way to ovarian steroid hormone stimulation. Enlarged and flattened morphological changes and actin rearrangement were also induced by the exposure of HDACi without ovarian steroid hormones. These results collectively suggest that histone acetylation is deeply involved in differentiation of EGCs together with up-regulation of glycodelin expression.

\section{Expression profiles of potential chemoattractants for peripheral blood CD16-negative natural killer cells in human endometrium}

Kotaro Kitaya, Nobue Daikoku, Takeshi Yamaguchi, Hideo Honjo

Kyoto Prefectural University of Medicine

Human endometrium is infiltrated by CD16-negative natural killer (NK) cells throughout the menstrual cycle. The number of endometrial CD16-negative NK cells is low in the proliferative phase, but acutely rises after ovulation, and peaks in the mid- to late secretory phase. Although the underlying mechanism explaining the postovulatory rise of endometrial CD16-negative NK cells remains yet unknown, selective recruitment from peripheral blood (PB) CD16-negative NK cells is a possible theory. We investigated the expression of the potential chemoattractants for PB CD16-negative NK cells in the human endometrium. Of chemokines examined. we found that macrophage inflammatory protein (MIP)-1beta, RANTES, monocyte chemoattractant protein (MCP)-2, MCP-3, MCP-4, 6Ckine, MIP-3beta, interferon-inducible protein (IP)-10, and monokine induced by gamma-interferon (Mig) were expressed, whereas MIP-1 alpha, interferon-inducible T-cell alpha-chemoattractant. and stromal cell-derived factor-1 were not. Although there were some differences in their expression patterns. their overall expression levels. especially in the vascular or perivascular area, were higher in the secretory phase than in the proliferative phase. Progesterone was a positive regulator of secretion of MIP-1beta, MCP-3, MCP-4. IP-10, and Mig from cultured endometrial stromal cells, but not of 6Ckine or MIP-3beta. By contrast. 17-beta estradiol had no significant effect on their secretion. These results support the idea that endometrial CD16-negative NK cells are recruited selectively from PB CD16-negative NK cells.

\section{The expression and effect of fibulin-1 during decidualization in human endometrial stromal cell induced by progesterone}

Tsuyoshi Nakamoto, Hidetaka Okada, Tatsuya Nakajima. Katsuhiko Yasuda. Hideharu Kanzaki

Kansai Medical University

Fibulin-1, an extracellular matrix as well as plasma glycoprotein, is distinct component of vessel walls. It was also found to inhibit cell motility and adhesion. We previously reported that progesterone stimulated the expression of fibulin-1 mRNA in endometrial stromal cells (ESCs). In the present study, we tried to clarify the spatial and temporal regulation of fibulin-1 by progesterone in human endometrium. Real-time PCR revealed a significant increase in fibulin-1 mRNA expression in ESCs treated with 6 a -methyl-17 a -hydroxy-progesterone acetate (MPA) or 8 bromoadenosine 3': 5'-cyclic monophosphate (8-Br-cAMP) compared with vehicle. Furthermore, MPA was a potent inducer of fibulin-1 mRNA expression in a dose dependent manner. Additionally. RU-486 almost completely inhibited MPA-induced fibulin-1 mRNA. On the other hand, $17 \beta$-estradiol (E2) alone did not increase fibulin-1 mRNA expression. To describe the menstrual cyclic regulation in vivo, real-time PCR and immunohistochemistry was performed in proliferative (Pro) and secretary (Sec) phases. Higher fibulin-1 mRNA expression was observed in Sec than in Pro phase. Fibulin-1 protein was expressed in glandular 
epithelia in Pro phase, but expression switched to the stroma in Sec phase. Small interfering RNA to inhibit fibulin-1 mRNA expression was added to ESCs culture, treated with MPA and 8-Br-cAMP, but no significant decrease of prolactin mRNA expression was observed. These results suggest that fiblin-1 is deeply involved in human endometrial decidualization induced by progesterone.

\section{Effects of Allograft Inflammatory Factor-1 (AIF-1) on cytokine productions in vitro study}

Ryutaro Nishida ${ }^{1}$, Shimada Shigeki ${ }^{1}$, Hideto Yamada ${ }^{1}$, Kazuya Iwabuchi $^{2}$, Emi Hirayama ${ }^{1}$, Mamoru Mirikawa ${ }^{1}$, Noriko Tsuruga ${ }^{1}$, Keiko Watano $^{2}$, Kazunori Onoe ${ }^{2}$, Hisanori Minakami ${ }^{1}$

${ }^{1}$ Depertment of Obstetrics and Gynecology, Hokkaido University Graguate School of Medicine

${ }^{2}$ Division of Immunobiology, Reseach Section of Pathophysiology, Institute for Genetic Medicine, Hokkaido University

Objective: We previously reported that the expression of AIF-1 mRNA in the mouse uterus diminished after insemination and increased in the peri-implantation period. The increased expressions of AIF-1. IFN- $y$, TNF- $a$ and NOS2 mRNA in poly(I:C)-treated mice were correlated with increasing rate of fetal resorption. In this study, we sought to investigate the interaction between AIF-1 and these molecules in vitro experiments.

Materials and Methods: 1) To analyze the function of AIF-1, transfection of the vectors containing mice AIF-1 cDNA into a macrophage cell line (RAW 264.7) was performed. Upon stimulation with bacterial lipopolysaccharide. we analyze the expression of IL-1 $\alpha$, IL-6, IL-10, IL-12p40, IL-12p70 mRNA and protein by RT-PCR and ELISA. respectively. 2) Another macrophage cell line (J774A.1) was stimulated with IFN- $\gamma$, and the expressions of AIF-1. NOS-2, TNF- a mRNA were analyzed by semi-quantitative RT-PCR. 3) After J774A.1, pre-treated with AIF-1 antisense oligonucleotide, was stimulated with IFN- $\gamma$, the expressions of NOS-2, TNF- a mRNA were analyzed.

Result: 1) The transfectants that overexpressed AIF-1 produced significantly large amount of IL-6, IL-10, and IL-12p40 but not IL-12p70 compared with control cells. The expression of IL-1 $a$ was not different between these cells. 2) The expressions of TNF- $a$, AIF-1, and NOS-2 mRNA were increased alternatively after the stimulation of IFN- $\gamma$. 3) The pre-treatment with AIF-1 antisense followed by the stimulation with IFN- $\gamma$ increased the expression of NOS-2 mRNA and prolonged that of TNF- a mRNA compared with control cells.

Conclusion: The overexpression of AIF-1 encouraged macrophages to produce IL-6, IL-10, and IL-12p40. The suppression of AIF- 1 resulted in the exaggerated NOS- 2 and TNF- $\alpha$ mRNA expressions. AIF-1 production in macrophages after insemination and in peri-implantation period might have the effect of inducing Th2 cytokines and suppressing NOS- 2 and TNF- $\alpha$, and thereby contribute to the maintenance of pregnancy.

\section{Increase of IUFD in pregnant mice immunized with massive cytokine producer TSST-1}

Eriko Takei ${ }^{1}$, Yosie Kametani ${ }^{2}$. Hidekazu Tamauchi ${ }^{1}$. Hiroko Maruyama ${ }^{1}$, Masakazu Ishikawa ${ }^{1}$, Sonoko Habu ${ }^{2}$ ${ }^{1}$ Kitasato University; ${ }^{2}$ Tohkai University

PROBLEM: Cytokines derived from the maternal immune response is currently considered to provide some effects on gestation. We examined fetal viability in pregnant mice immunized with Toxic shock syndrome toxin-1 (TSST-1), which induces rapid and massive cytokine production as bacterial super antigen, using TCR transgenic (TCR-Tg) mice with TCR-V $\beta 15$.

METHOD: Pregnant mice were immunized with TSST-1 on 17 gestation days and then submitted for measuring serum IL-2 and intra uterine fetal death (IUFD) and for histological analysis of placenta status on 18 days.

RESULTS: IUFD was increased with the apoptotic placentas in the immunized mice only if mother was TCR-Tg. However, if mother is non-TCR-Tg and father is TCR-Tg, IUFD was low. In consistent with the increased IUFD. serum IL-2 was higher in immunized TCR-Tg pregnant mice than non-TCR-Tg mice.

CONCLUSIONS: Our results suggest that massive IL-2 production in pregnant mice immunized with 
TSST-1 may result in high IUFD through apoptosis of placentas.

\section{Regulatory $T$ cells play a very important role for maintenance of allogeneic pregnancy}

Yasushi Sasaki. Satomi Miyazaki, Subaru Higuma, Masatoshi Sakai, Shigeru Saito

Toyama medical and pharmaceutical university

Purpose: A immunosuppression or tolerance is important to maintenance for pregnancy. Recent data showed that regulatory $\mathrm{CD}^{+} \mathrm{T}$ cells express IL-2 receptor a (CD25) on their surface and suppress the immune responses of T cells through CTLA-4 molecule. We reported that regulatory T cells increased in earlypregnancy decidua, while Treg ratio in spontaneous abortion cases did not increase. (Y Sasaki, Mol Hum Reprod 2004; 10:347-353) These results suggest the increased regulatory $T$ cells in the decidua play some roles for maintenance of pregnancy by regulating maternal cytotoxic $\mathrm{T}$ cells. In this study, we investigate whether the administration of anti mouse CD25 monoclonal antibody(PC61) induce abortion in early pregnant mouse.

Materials \& Methods: BALB/C male and C57BL/6 female mouse were crossbred and pregnant mouse was given intraperitoneal injection of anti-CD25 mAb $(0.5 \mathrm{mg} / \mathrm{body} \times 2)$ on the 4 th and 7 th day of gestation. Control mouse was given saline as a control. Then the mice were sacrificed on day 11 th and examined the number of viable and aborted fetuses.

Results: 1. When anti-CD25 mAb was injected to pregnant $\mathrm{BALB} / \mathrm{C}$ mice. $\mathrm{CD} 4{ }^{+} \mathrm{CD} 25^{+}$cells disappeared on day 11 th of gestation. 2. There was no differences between the number of total fetuses of anti-CD25 mAb injected groups and that of control groups. On the other hand, abortion ratio of anti-CD25 mAb group was significantly higher than that of control group $(37.6 \pm 39.6 \%$ vs. $0 \%, \mathrm{p}=0.0441)$. In allogenic mice. inhibition of IL-2 receptor $\alpha$ induced abortion, however this $\mathrm{mAb}$ treatment did not affect the abortion rates in syngenic pregnancy.

Conclusion: Regulatory $\mathrm{T}$ cells play an important role for protection of maternal allogeneic rejection.

\section{G-CSF inhibits IFN-gamma production of NKT cells through suppressor of cytokine 1signaling}

Yoshiaki Ohta ${ }^{1}$, Satoshi Hayakawa ${ }^{2}$, Ikuko Ohta ${ }^{1}$, Kenji Sugita $^{1}$. Fumihisa Chishima ${ }^{1}$. Tatsuo Yamamoto ${ }^{1}$. Rie Kawaguchi $^{3}$, Tadao Tanaka ${ }^{3}$

${ }^{1}$ Department of Obstetrics and Gynaecology, Nihon University School of Medicine

${ }^{2}$ Department of Infectious Disease Control , Nihon University Advanced Medical Research Center

${ }^{3}$ Department of Obstetrics and Gynaecology; Jikei University School of Medicine

Purpose and backgrounds: One of the mechanisms of leukocytosis during pregnancy is attributed to G-CSF of placental origin. Aside from its stimulatory effects on leukocyte progenitors, we have reported novel suppressive roles of G-CSF on proinflammatory cytokine induced IFN-gamma production of decidual large granular lymphocytes. In this study, we examined effects of G-CSF on suppressor of cytokine signaling (SOCS) molecules on established LGL cell lines.

Materials and Methods: Large granular lymphocyte cell lines, KHYG-1 (CD56 $\left.{ }^{+} \mathrm{CD} 3^{\mathrm{dim}} \mathrm{CD} 16^{\circ}\right)$ and MOTN $\left(\mathrm{CD} 56^{+} \mathrm{CD} 3^{-} \mathrm{CD} 16^{-}\right)$were employed for following studies. Cells were maintained in the presence or absence of IL-2 $(1000 \mathrm{IU} / \mathrm{ml})+\mathrm{IL}-12(100 \mathrm{IU} / \mathrm{ml})$ for activation. Various concentrations of human recombinant G-CSF were added for these cultures. IFN-gamma producing cells were estimated by ELISPOT. We examined SOCS mRNA levels by semi-quantitative RT-PCR.

Results: Both MOTN (NK) and KHYG-1 (NKT) produced IFN-gamma in the presence of IL-2 and IL-12 up-regulated IFN-gamma production in these cells. G-CSF suppressed IFN-gamma production in KHYG-1 cells in a dose dependent manner but did not affect production in MOTN. High concentration of G-CSF (over 25000 $\mathrm{ng} / \mathrm{ml}$ ) suppressed mRNA expression of SOCS-1. 2, 3 in KHYG-1.

Conclusion: Our results suggest possible involvement of SOCS-1, 2, 3 in G-CSF induced suppression of IFN-gamma production in NKT cells. 
19. Expression of indoleamine 2,3-dioxygenase (IDO) in human pregnancy peripheral blood- and decidual monocytes and dendritic cells supports maternal-fetal tolerance. $<$ br $><$ br $>$

Naoko Miwa ${ }^{1}$, Satomi Miyazaki ${ }^{1}$, Satoshi Hayakawa ${ }^{2}$, Rie Kawaguchi ${ }^{3}$, Subäru Higuma ${ }^{1}$, Yasushi Sasaki ${ }^{1}$, Masatoshi Sakai ${ }^{1}$, Saito Shigeru ${ }^{1}$

${ }^{1}$ Obstetrics and gynecology, Toyama Medical and Phermaceutical University; ${ }^{2}$ Department of Infectious Disease Control and Clinical Immunology, Nihon University Advanced Medical Reserch; ${ }^{3}$ Obstetrics and gynecology, Jikei Medical School

Recent reports have suggested that the enzyme indoleamine 2,3-dioxygenase (IDO) activity in the human placenta is high and this enzyme play an important role for maintenance of pregnancy. The antigen-presenting cells, macrophages (Mf) and dendritic cells (DC), also express IDO. IDO expression is induced by IFNg and CTLA-4 expressed on regulatory T cells. In this study, we have studied the expression of IDO in Mf and DC in human pregnancy peripheral blood and early pregnancy decidua. Samples were collected from non-pregnant controls $(n=7)$, pregnant women $(n=12)$, induced abortion cases $(n=5)$, and spontaneous abortion cases $(n=4)$. Mononuclear cells from these samples were incubated for $24 \mathrm{~h}$ with or without stimulation by IFNg or recombinant human CTLA-4/Fc chimera and then analyzed the percentage of IDO positive Mf or DC by flow cytometry. In un-stimulated condition, the expressions of IDO in decidual Mf and DC were increased compare to those in peripheral blood, and these expressions of decidual Mf and DC of spontaneous abortion cases were significantly lower compare to those in normal pregnancy subjects $(1.0 \pm 0.9 \%$ vs $7.0 \pm 5.9 \%, 0.8 \pm 0.8 \%$ vs $3.2 \pm 1.5 \%$, respectively) $(\mathrm{p}<0.05)$. After the stimulation, IDO expressions in $\mathrm{Mf}$ and DC were elevated. IFNg mainly augmented the expression of IDO in Mf, whereas CTLA-4 Ig/Fc mainly augmented in DC. IDO expression rates of peripheral blood $\mathrm{Mf}$ after the stimulation were significantly higher in first and third trimester pregnant subjects than those in non-pregnancy subjects. These results suggest that in pregnancy peripheral blood, Mf could change into an inhibitory type of the antigen-presenting cells. IDO expressions in decidual Mf and DC were elevated in normal pregnancy and those in spontaneous abortion cases were decreased suggesting that IDO plays some important role for maintenance of pregnancy.

\section{CYTOKINES PROFILE OF NATURAL KILLER CELLS IN THE HUMAN EARLY PREGNANCY}

Satomi Miyazaki, Subaru Higuma, Yasushi Sasaki, Sakai Masatoshi, Sigeru Saito

Toyama Medical and Pharmaceutical University.

INTRODUCTION: The Th1/Th2 paradigm was evolved by the identification of Tr1 (IL-10>TGF- $\beta$ ) and Th3 (TGF- $\beta<\mathrm{IL}-10$ ). NK cells are classified into NK1 and NK2 subsets like T cells, but NK3 and NKr1 subset have never been reported. In this study, we investigated the production of TGF- $\beta$, IL- 10, IFN- $\gamma$, TNF$a$. IL-4, IL-5 or IL-13 from the peripheral blood and decidual CD56 $6^{\text {bright }}$ NK cells and CD56 ${ }^{\mathrm{dim}}$ NK cells in non-pregnant healthy women $(n=17)$, early normal pregnant women $(n=17)$ and abortion cases $(n=10)$ by flow cytometry and examined whether the Th1/Th2/Th3/Tr1 paradigm was adapted to NK cells.

RESULTS: In the peripheral blood of non-pregnant subjects, the main populations of CD56 ${ }^{\text {bright }}$ NK cells and $C D 56^{\text {dim }}$ NK cells were IFN- $\gamma$ producing cells. IFN- $\gamma$ producing NK cells did not produce type 2 cytokines, IL-10 and TGF- $\beta$. After pregnancy. IL-10 producing peripheral blood CD56 ${ }^{\text {bright }}$ cells and CD56 ${ }^{\mathrm{dim}}$ cells were significantly higher than those in non-pregnant subjects. They did not produce type 1 cytokines, type 2 cytokines and TGF- $\beta$ indicating that they are classified into NKrl cells. In abortion cases, NKr1 cells were decreased to non-pregnancy level. In the early pregnancy decidua, the main populations of CD56 ${ }^{\text {bright }}$ NK cells and CD56 ${ }^{\mathrm{dim}}$ NK cells were TGF- $\beta^{+}$. IFN- $\gamma^{-}$. IL-4, IL-5; IL-10- NK3 cells. In abortion cases, NK3 cells were significantly lower than those in normal pregnant subjects. On the other hand. IFN- $\gamma$ producing decidual CD56 ${ }^{\mathrm{dim}} \mathrm{NK}$ cells significantly increased in abortion cases.

CONCLUSION: NK cells can be classified into NK1, NK2, NK3 and NKr1 cells. In human pregnancy. 
$\mathrm{NKr} 1$ cells are the main population of peripheral blood and NK3 cells are the main population of decidual lymphocytes. These data suggested that NKrl cells and NK3 cells might play some important roles for maintenance of pregnancy by regulation of maternal immune function.

\section{Effect of lipopolysaccharide (LPS) and tumor necrosis factor- $\alpha$ (TNF- $\alpha$ ) on activin secretion in primary amniotic epithelial cell cultures}

Yumiko Abe, Hiromitsu Shinozaki, Takeshi Takagi, Masahiro Ito, Takashi Minegishi

Gunma University

Background of study: Growth factors and enzymes produced in amniotic epithelial cells participate in maintaining the strength of fetal membrane. Activin is a growth factor regulating cell proliferation and differentiation and is produced in many types of cells including amniotic epithelial cells. It has been reported recently that hypoxemia increased activin levels in systemic circulation and local activin levels were elevated in rheumatoid arthritis.

Objective: To study the effect of lipopolysaccharide (LPS), a gram negative bacterial cell wall component, and inflammatory cytokine tumor necrosis factor- $a$ (TNF- $a$ ) on activin secretion from cultured amniotic epithelial cells. Methods: With the permission of The Internal Review Board and the informed consent of the patients, we obtained a fetal membrane sample during a cesarean section, from a patient without systemic disease, sign of premature delivery or fetal complications. Amniotic epithelial cells were dispersed by trypsin and cultured in DME/Ham's F12 medium supplemented with 10\% FBS.

Results: 1) Both LPS (3-30 $\mu \mathrm{g} / \mathrm{ml})$ and TNF- a $(3-30 \mathrm{ng} / \mathrm{ml})$ decreased BrdU incorporation in a dose dependent manner at 96 hours culture. 2) Activin A secretion was stimulated by both LPS $(3-30 \mu \mathrm{g} / \mathrm{ml})$ and TNF- a $(3-30 \mathrm{ng} / \mathrm{ml})$ in a dose dependent manner. After 96 hours culture, activin A concentrations in the medium of cells with vehicle (control). LPS $(30 \mu \mathrm{g} / \mathrm{ml})$ and TNF- a $(30 \mathrm{ng} / \mathrm{ml})$ were $408 \pm 21 \mathrm{pg} / \mathrm{ml}, 669 \pm 60$ $\mathrm{pg} / \mathrm{ml}, 1944 \pm 62 \mathrm{pg} / \mathrm{ml}($ Mean \pm SE, $\mathrm{n}=4)$, respectively, and significantly higher in LPS and TNF- $\alpha$ treated cells, compared to the control $(P<0.01)$. 3) Activin A secretion was stimulated by LPS $(3-30 \mu \mathrm{g} / \mathrm{ml})$ and TNF- $a$ $(3-30 \mathrm{ng} / \mathrm{ml})$ in a dose dependent manner. 4) A co-treatment of anti TNF- $a$ antibody and TNF- $a$ suppressed the increase of activin A secretion brought about by TNF- $a$.

Conclusion: The increased secretion of activin A by LPS and TNF- a was observed in primary amniotic epithelial cell culture. It was deduced that local activin A levels also increase in chorioamnionitis.

\section{Detection of HLA-F expression on placental trophoblasts in various stages of pregnancy - Collaboration of HLA-E, -F, and -G- \\ Takanori Shobu ${ }^{1,2}$, Noriko Sageshima ${ }^{1}$, Mai Tomiyama ${ }^{1}$, Takeaki Okamoto' ${ }^{1}$ Yukio Hayashi ${ }^{2}$, Katsuhiko Hatake ${ }^{1}$. Daniel E. Geraghty ${ }^{3}$. Akiko Ishitani ${ }^{1}$ \\ ${ }^{1}$ Department of Legal Medicine, Nara Medical University; ${ }^{2}$ Hayashi Women's Clinic, Nara; ${ }^{3}$ Fred Hutchinson Cancer Research Center, Seattle, USA}

OBJECTIVE: We have tested HLA-F expression on a number of cell lines and found intracellular expression on several lines using monoclonal antibodies we had developed, but surface expression on only EBV-transformed B cells. Previously it had been thought that normal cells did not express HLA-F on the cell surface. To further understand HLA-F function of pregnancy. we investigated the expression of HLA-F on trophoblasts in each stage (first. second. and third trimester) including the expression of HLA-E and -G.

METHODS: Immunohistochemical stainning was performed with anti HLA-E, -F, and -G antibodies. To identify cells where HLA-F could be detected, immunohistochemical double staining was performed with anti HLA-F antibody and anti cytokeratin or anti vimentin antibodies using fluorescent staining.

RESULTS: As previously shown, we detected HLA-G expression on extravillous trophoblasts, and found HLA-E expressed weakly on all trophoblasts in all stages. Weak expression of HLA-F was found on all types of 
trophoblasts, however HLA-F was expressed strongly on the extravillous trophoblasts that had invaded the maternal decidua.

CONCLUSION: We found that HLA-F is expressed not only on the villous trophoblasts but also on the extravillous trophoblasts in all stages of pregnancy. We speculate that HLA-E, -F, and -G together may collaborate in the protection of fetus from maternal immune cells.

23. A prospective study of antiphospholipid antibody screening for pregnant women: prevalence and subsequent pregnancy outcomes

Noriko Tsuruga ${ }^{1}$, Hideto Yamada ${ }^{1}$, Emi Hirayama ${ }^{1}$, Itsuko Furuta ${ }^{1}$, Gen Kobashi ${ }^{1}$, Tatsuya Atsumi ${ }^{1}$, Hitoshi Okubo $^{2}$, Noriaki Sakuragi ${ }^{1}$, Hisanori Minakami ${ }^{1}$

${ }^{1}$ Hokkaido University School of Medicine; ${ }^{2}$ Sapporo Maternity Womens' Hospital

Objectives: By prospective study, to determine the prevalence of a variety of antiphospholipid antibody (APL) and to evaluate pregnancy outcomes of women with APL.

Methods: The eight hundred forty-one pregnant women in the first trimester were enrolled in this study. Six kinds of APLs included lupus anticoagulant (LA), anticardiolipin (aCL) $\operatorname{IgG} / \operatorname{IgM} / \operatorname{IgA}$, and anti-prothrombin-phosphatidylserine (aPS/ PT) IgG/IgM. Among them. 509 women had completed their deliveries and were evaluated whether the presence of APLs was related to the pregnancy outcome such as premature delivery, gestosis, pregnancy induced hypertension (PIH), and intrauterine fetal growth restriction (IUGR).

Results: The frequency of APL was found to be $9.6 \%$. The frequencies of each APL were as follows; aCL $\operatorname{IgG}(2.0 \%), \operatorname{IgM}(0 \%), \operatorname{IgA}(0.6 \%), \mathrm{aPS} / \mathrm{PT} \operatorname{IgG}(0.4 \%)$. IgM $(7.3 \%)$. LA $(0.8 \%)$. The presence of LA was found to be associated with gestosis (RR 10.0, 95\% CI 2.2-46.2) and PIH (RR 9.6, 95\% CI 1.0-91.0). Additionally, the presence of two or more APLs was associated with PIH (RR 9.6, 95\%CI 1.0-91.0). Neither of APL was associated with premature delivery nor IUGR.

Conclusions: In this prospective study, we demonstrated the prevalence of APL among pregnant women. We for the first time demonstrated that the presence of LA and two or more APL were significant risk factors of gestosis and PIH.

\section{Decreased serum levels of macrophage migration inhibitory factor in miscarriages with normal chromosome karyotype}

Hideto Yamada, Emi Hirayama, Mamoru Morikawa. Shigeki Shimada, Jun Nishihira, Hisanori Minakami Hokkaido University

BACKGROUND: The aim of this study was to determine serum concentrations of macrophage migration inhibitory factor (MIF) during normal pregnancies, and to assess whether serum MIF concentrations early in pregnancies predict the subsequent outcome in women with recurrent miscarriage (RM).

METHODS: Serum MIF concentrations were measured by ELISA methods. Sera were collected from normal women in first (Group I, $\mathrm{n}=29$ ), second (Group II, $\mathrm{n}=25$ ), third trimester (Group III, $\mathrm{n}=26$ ). and from 78 RM women at 4-6 weeks of gestation. Eleven of these 78 pregnancies subsequently ended in first trimester miscarriage with normal fetal chromosome karyotype (MsNK), 7 ended in first trimester miscarriage with abnormal karyotype (MsAK), and 3 ended in biochemical pregnancy. The other 57 pregnancies ended in live birth ( $\mathrm{Lb}$ ) between $32 \mathrm{nd}$ and 41 st week of gestation.

RESULTS: Median MIF concentrations in Group I. II, and III were 17.6. 16.4. and $15.1 \mathrm{ng} / \mathrm{ml}$, respectively. MIF concentrations during early gestation in RM women with subsequent MsNK. MsAK, and Lb were 8.1. 11.4, and $16.4 \mathrm{ng} / \mathrm{ml}$, respectively. MIF concentrations in RM women with MsNK were significantly lower than those in RM women with $\mathrm{Lb}$ and than those in Group I. II. and III.

CONCLUSIONS: Decreased serum MIF concentrations during early gestation were found in RM women 
with MsNK, and might be related to etiology of miscarriage.

\section{Prevalence of diverse antiphospholipid antibodies in women with recurrent spontaneous abortion} Shigeki Shimada. Tatsuya Atsumi. Emi Hirayama, Mamoru Morikawa, Hisanori Minakami. Hideto Yamada Hokkaido University

OBJECTIVES: It remains uncertain what combination of antiphospholipid antibodies is clinically valid in screening of recurrent spontaneous abortion (RSA). We sought to determine the prevalence of various antiphospholipid antibodies in women with RSA.

METHODS OF STUDY: Between 1997 and 2002, 114 consecutively seen women with RSA were recruited. All women were not currently pregnant, and all gave informed consent to participate. Blood samples were obtained, and lupus anticoagulant (LA) and anticardiolipin B2-glycoprotein I (aCLB2GPI) IgG were measured. Sera were then separated and stored at $-80^{\circ} \mathrm{C}$ until use. aCLB2GPI IgM and IgA, anticardiolipin (aCL) IgG, IgM and IgA, antiphosphatidylserine prothrombin (aPS-PT) IgG and IgM, and antiphosphatidylethanolamine (aPE) IgG and IgM, were measured reirospectively.

RESULTS: Using the standard combinations of antiphospholipid antibody measurements in screening for RSA as definitional: LA plus aCLB2GPI IgG and IgM, and LA plus aCL IgG and IgM. $2.6 \%$ and $4.4 \%$ of women with RSA had the antiphospholipid antibody syndrome, respectively. When aCLB2GPI IgA, aCL IgA. and aPS-PT IgG and IgM were combined with the standard antiphospholipid antibody measurements. the proportion of patients positive for antiphospholipid antibody reached $7.0 \%$. When aPE $\operatorname{IgG}$ and $\operatorname{IgM}$, were included, the proportion increased to $26.3 \%$ ( 30 of 114 women). Women with midtrimester fetal losses presented with a significantly higher prevalence of LA, aCLB2GPI IgG/IgA, aCL IgG, and aPS-PT IgG. but not aPE, than did women with early recurrent abortions. Women with 2 consecutive abortions presented with a higher prevalence of antiphospholipid antibodies than did women with 3 or more abortions.

CONCLUSIONS: We demonstrated the prevalence of a wide variety of antiphospholipid antibodies in women with RSA. This information will aid clinical practice in screening of RSA.

\section{Anti-factor XII Antibodies in Patients with Recurrent Pregnancy Losses Recognize the Amino-Terminal Heavy Chain Region of Factor XII}

Akifumi Inomo, Toshitaka Sugi, Tsunehisa Makino

Tokai University

Objective: Recently, surprisingly high prevalence of factor XII deficiency among patients with thrombosis and RPL have been reported. Since the presence of antibodies to factor XII in patients with lupus anticoagulant has been reported, we have now tested plasma samples from RPL patients for the presence of autoantibodies to factor XII and for the epitope mapping of factor XII recognized by those autoantibodies.

Methods and Results: One hundred ninety one patients with RPL were screened for factor XII activity. We found $34(17.8 \%)$ patients with factor XII deficiency. We hypothesized that factor XII deficiency may be partly due to presence of antibodies to factor XII and tested plasma samples from patients with factor XII deficiency for the presence of antibodies to factor XII by immunoblot and ELISA. We found that $53.5 \%$ of factor XII deficient patients with RPL were positive for IgG anti-factor XII antibodies $(p<0.001)$. All anti-factor XII antibodies recognized the heavy chain of factor XII. Several peptides were synthesized after consideration of the structure of factor XII based on literatures. and we found that anti-factor XII antibodies recognized the amino-terminal heavy chain region of factor XII.

Conclusions: Literatures have reported that human factor XII binding to the glycoprotein Ib-IX-V complex via the amino-terminal heavy chain region inhibits thorombin-induced platelet aggregation. We confirmed that patients with factor XII deficiency had antibodies to factor XII and those autoantibodies recognized the heavy chain of factor XII, especially the amino-terminal sequences. Therefore. we hypothesize that autoantibodies to 
factor XII may be associated with diseases, such as thrombophilia and RPL.

27. Impaired vascular function in women with recurrent pregnancy loss: Involvement of antiphospholipid antibodies

Mikiya Nakatsuka, Soichi Noguchi. Yasuhiko Kamada, Chebib Chekir, Aiko Sasaki, Lin Hao, Yuji Hiramatsu Okayama University

The causes of recurrent pregnancy loss (RPL) are classified as genetic, anatomic, endocrinologic, immunologic, microbiologic, and environmental. However, vascular changes associated with RPL remain poorly studied.

28. Study on clonally expanded $T$ cells before and after husband's lymphocyte immunotherapy in peripheral blood of patients with habitual abortion

Takaaki Kariya ${ }^{1}$, Kazuhiko Hayashi ${ }^{1}$. Bunpei Ishiduka ${ }^{2}$

${ }^{1}$ St Marianna University, Yokohama City Seibu Hospital; ${ }^{2}$ St Marianna University

Purpose: Although the pathogenesis of habitual abortion (HA) has not been established, there is some evidence to indicate the involvement of various immunological factors. Clinically, this view is perhaps supported by reports indicating that husband's lymphocyte immunotherapy is often used to improve fertility of patients with HA, which probably acts through immune-mediated mechanisms.

Methods: By using RT-PCR-SSCP analysis, we first investigate the peripheral T cell clonality in healthy women. Next we compared the T cell clonality in HA, before and after husband's lymphocyte immunotherapy. Similar analysis was done about the examples of one or two times addition of immunotherapy and after delivery. Decidual analysis of two cases. early missed abortion and artificial abortion, was done. Particular TCR BV gene products of accumulationg $\mathrm{T}$ cell were determined by direct sequencing.

Results: Although peripheral $\mathrm{T}$ cells in healthy women were extremely heterogeneous, non-pregnancy patients with a history of HA before immunotherapy were not. It was also revealed, however, that immunotherapy reduced the number of accumulation $T$ cell clones. Accumulating $T$ cell clones levels of examples of one or two times addition of immunotherapy and after delivery were similar to post-immunotherapy of HA. We determined amino acid sequences of T cell clones accumulating in TCR BV1 gene products of the RT-PCR. Dominant accumulating clone at a frequency of $43.5 \%$ in the BV1 before immunotherapy decreased to $3.1 \%$ after that therapy.

Conclusion: Our results, together with the clinical effectiveness of immunotherapy, suggest that accumulation of $\mathrm{T}$ cell clonotypes, which probably resulted from antigenic stimulation, is involved in the pathogenesis of HA. These findings enhance our understanding of the mechanisms by which immunotherapy might prevent rejection of the fetus in HA.

\section{Role of Cathepsins and Cystatins in patients with Recurrent Miscarriage}

Tamao Nakanishi ${ }^{1}$, Yasuhiko Ozaki ${ }^{1}$, Kyoko Nozawa ${ }^{2}$, Mayumi Sugiura ${ }^{1}$, Kaoru Suzumori ${ }^{1}$

${ }^{1}$ Nagoya City University; ${ }^{2}$ Nagoya Johoku municipal hospital

During implantation, trophoblasts penetrate the maternal decidua by secreting proteases. It has been reported that cathepsins are highly expressed in mouse villi, and play an important role in normal embryonal growth and decidualization. In this study, we evaluated the levels of cathepsins and their endogenous inhibitors, cystatins in endometrial tissue and serum of patients with recurrent miscarriage (RM).

Decidual and villous tissue was surgically collected from $22 \mathrm{RM}$ patients and 12 healthy women. Immunohistochemistry (IHC), SDS-PAGE and western blotting analysis were performed using antibodies against cystatins and cathepsins. The concentrations of cystatins and cathepsins in those tissues were measured by ELISA. In addition. we measured the cystatin C levels in serum from 85 Japanese women with RM and 32 
healthy women without history of miscarriage.

IHC stainings of cathepsin B. D. H, K, L, S and cystatin C were observed in the cytoplasm of epithelial cells in the decidua. Western blotting analysis supported the presence of cystatin $\mathrm{C}$. The concentrations of cathepsin D. H and S in RM decidua were significantly higher than in controls, as judged by ELISA. The serum levels of cystatin $C$ were significantly lower in the RM group than in the control group $(p=0.0024)$.

Our findings suggest that regulation of the cathepsin-cystatin system may play an important role in RM.

\section{Recurrent spontaneous abortion with antiphospholipid antibodies; Incidence and therapeutic outcome}

Rie Kawaguchi, Akemi Murao, Maho Ozawa. Yoshiaki Akiyama, Tadao Tanaka

Jikei University School of Medicine

Introduction: antiphospholipid antibodies (APA). especially the IgG subtype, have been associated with thrombotic and cytotoxic events of placental vessels and trophoblast, which could lead to pregnancy loss, but there have been a few reports about IgM subtype. Moreover, therapeutic principle to the patients of recurrent spontaneous abortion (RSA) with APSs have nor been fully clarified.

Objectives: 1) To investigate the incidence of $\operatorname{IgG}$ and $\operatorname{IgM}$ subtypes of APSs including anti-cardiolipin (CL), anti-phosphatydil ethanolamine (PE), and anti-phosphatydil serin (PS) in patients with RSA. 2) To explore to effective therapy for RSA with these APAs. $<$ br $>$ Method: 141 women with history of RSA registered at Jikei University Hospital were examined retrospectively. 1) Prior to treat and conceive. APAs were detected. 2) Patients with any of these APAs were treated with herb-medicine, aspirin and/or heparin.

Results: 1) Incidence of antibodies to CL-IgG. IgM, PE-IgG, IgM, and PS- IgG. IgM were 17/163 (10.4\%). 40/164 (24.4\%), 28/150 (18.7\%), 4/141 (2.8\%). 7/132 (5.3\%), 37/127 (29.1\%), respectively. Positive antibodies (IgG-group) were recognized in 40/174 (22.9\%), while IgM antibodies (IgM-group) were 60/170 (35.3\%) for total samples. 2) Pregnancy was successfully maintained in 60/68 (88.2\%) of RSA with APAs. In IgG group. $25 / 29(86.25)$ were successful, and 35/39 (89.7\%) in IgM-group.

Conclusions: 1) APAs were greatly involved in RSA, and significantly higher incidence was found in PE-IgG. CL-IgM and PS-IgM antibodies than in other APAs. And the incidence of IgM antibodies was significantly higher than that of IgG. 2) Anticoagulation-therapy to RSA patients with any of APAs was effective. obtaining $88.2 \%$ of the high production in successful pregnancy, and the subtypes of APAs did not influence the therapeutic outcome.

\section{Development of human graffian follicle following xenotransplantation into immunodificient mice}

Tomoko Yoshimoto, Yukihiro Terada, Takashi Murakami. Kunihiro Okamura

The University of Tohoku

BACKGROUND: There are some reports regarding follicular growth following xenograftic transplantation of human ovarian cortex into immunodeficient mice. We studied human follicular growth and immunohistochemical expression of steroidgenic enzymes in the graft of non-pathorogical human ovary following xenograftic transplantation into non-obese diabetic immune deficient (NOD-SCID) mice and NOD/SCID/g cnull (NOG) mice.

METHODS: Human ovarian tissue were transplanted into back skin, kidney capsule and ovarian bursa of NOD-SCID or NOG mice. After xenotransplantation and gonadotropin stimulation. the number of developing follicle and expression of steroidogenic enzymes (P450 choresterol side chain cleavage (P450scc), 3 beta-hydroxysteroid dehydrogenase ( 3 beta-HSD). cytochrome P450 alpha-hydroxyiase (P450c17). cytochrome P450 aromatase (P450arom)). were studied by immunohistchemistry.

RESULTS: The number of antral follicle in human ovarian graft following transplantation/stimulation into ovarian bursa of NOD-SCID mice were 28 in 56 grafts. However. only one antral follicle was developed in 12 grafts following transplantation into mice back skin. In NOG mice. 23 antral follicle developed in 53 
human ovarian grafts, and among these, graffian follicle development was observed in 2 human ovarian grafts. Expression of steroidgenic enzymes were observed in preantral/antral human follicle. Further, P450arom immunostain was positive in granulosa cells of human grafiian follicle developed in NOG mouse.

CONCLUSIONS These findings suggest that expression of steroidogenic enzymes in human follicles following xenograftic transplantation into NOD-SCID mice, was similar to that of non pathorogical human ovaries. Further, immune condition in host mice and transplantation site of graft are crucial for successful rate in xenografting of human ovarian cortex.

\section{Isolation of undifferentiated cells derived from embryos reconstituted by somatic cell nuclear transfer} in the rabbit

Yorino Sato, Ai Kazami, Naoki Okamoto, Koji Hosaka, Kahei Sato

Departoment of Applied Life Science, Graduate School of Bioresorce Sciences, Nihon Unversity

Embryonic stem cells (ES cells) will be useful in the regenerative medicine. However, there are problems of immunorejection to apply the cells in clinical. Further, rabbits are valuable as surgical disease model for human. In this study, we investigated to produce reconstituted embryos derived from somatic cell nuclei, and to isolate undifferentiated cells from the rabbit embryos.

Mature Japanese White female rabbits were superovulated with HMG and hCG. Cumulus-oosyte complexes were obtained from oviducts, and cumulus cells were removed by hyaluronidase and pippeting. Nuclear donor cells were fibroblasts gathered from abdominal skin of Dutch strain rabbits. MII oocytes were enucleated in modified HTF medium containing cytochalasin B. The chromosome removal was confirmed by staining with Hoechst 33342 under ultraviolet light. The reconstituted embryos were produced by two methods. electorofusion and microinjection. In the electorofusion methods, a fibroblast was inserted into the perivitelline space of the enucleated oocyte using injection pipette. The pairs were induced of the cell membrane fusion using the cell fusion apparatus. After electrofusion, these oocytes were cultured for 1 hour in HTF medium. On the other hand, in microinjection methods, a single donor cell was drowning in and out of the injection pipette until the plasma membrane was broken. After, the nuclei were injected individually into enucleated ooplasm. The reconstituted oocytes were activated with $7 \%$ ethanol for 5 minutes, and exposed to 6-dimethylaminopurine (DMAP) and Cycloheximide (CHX) in HTF medium for 3 hours at room temperature to inhibit the chromosome condensation and the release of 2 nd polar body. The embryos developed to blastocyst were cultured on the feeder cells layer in Ham-F12 medium.

The number of reconstituted embryos treated with DMAP and CHX was 72. The rate of cleavage to more than 8 -cells stage was $38.8 \%$. The developmental rate to blastocyst was low comparison with the control, however some embryos were confirmed development to hatching or more stage in vitro and attached on the feeder cells layer.

From the results obtained, we confirmed in vitro development to hatching and more stage of reconstituted embryos in the rabbit, and obtained attached cell population from the embryos. Further, there was no difference of attached cell populations comparison with control in morphology. It is suggest that isolation of undifferentiated cells will be possible. However, developmental rate was significantly lower than the control. It is necessary to improve culture conditions for effective development of reconstituted embryos.

\section{Development of Reconstructed Embryos $<$ br $>$ by Nuclear Transfer Using Mouse Somatic Cells}

Naoki Okamoto. Ai Kazami, Yorino Sato, kouji Hosaka, Kahei Sato

Department of Applied Life Science, Nihon University Graduate School of Bioresource Sciences

Object: Cloning entire animals from somatic cells has recently been accomplished in the sheep, mouse. cattle, pig, and goat, Cloning has been produced individuals such that the offspring have an essentially identical genome. Mammalian cloning has been achieved by introducing the nucleus of a donor cell into an enucleated 


\section{Abstracts of the 19th Annual Meeting}

oocyte. Recently low rate of development have been suggested in reconstruction of mice embryo by nuclear transfer. In this study reconstructed oocytes that nuclear transfer using $\mathrm{G} 0 / \mathrm{Gl}$-phase donor fetal fibroblasts ( $\mathrm{Fb}$ ). adult fibroblasts and cumulus cell, compare with respective development. Objective was a lift developmental capacity.

Method: Cumulus-oocyte complexes were harvested from superovulated B6D2F1 (C57BL/6 $\times$ DBA/2) mouse and treated with hyaluronidase to remove cumulus cells in modified-HTF (mHTF) medium. Oocyte were enucleated and injected with the fibroblasts derived from 14-16h serum-starved cells. These embryos were induced fusion by electric stimulation. Unless oocytes were enucleated and injected with the cumulus cell nuclei. Reconstructed embryos were activated by $7 \% \mathrm{EtOH}$ or strontium. Subsequently, the embryos were cultured in vitro, and their developmental capacities were evaluated.

Results: The results demonstrated that $5 \%, 25 \%, 36 \%$ of reconstructed embryos cleaved, respectively. These results demonstrate that donor of fetal $\mathrm{Fb}$ cells and cumulus cell can be developed more efficient than adult $\mathrm{Fb}$ cells. Farther, from our results it was indicated that a microinjection method was better than an electrofusion method for transfer of somatic cell nuclei.

\section{Developmental Ability of Mouse Oocyte Derived from Maturation Culture in Vitro}

Ryoko Tomiyama ${ }^{1}$, Jyunko Sunaga ${ }^{2}$, Yayoi Nishi ${ }^{1}$, Michiko Oouchi ${ }^{2}$, Ayaka Katsumata ${ }^{2}$, Misao Satomi ${ }^{1}$, Takashi Abe $^{1}$, Shigeo Akira ${ }^{1}$, Kahei Sato ${ }^{2}$, Toshiyuki Takeshita ${ }^{1}$

${ }^{1}$ Nippon Medical School; ${ }^{2}$ Dept. Bioresouce Sciences, Nihon University

In domestic animals and laboratory animals, immature oocytes from ovaries have a potentiality for mature under the in vitro culture and develop to live pups following in vitro fertilization and embryo transfer. However. there is higher incidence of embryo/fetal losses in the embryos derived from in vitro matured oocytes. compared to ovulated or in vivo matured oocytes. This study was carried out to confirm when the embryo/fetal losses were occurred through their embryonic development.

Sexually matured ICR female mice were induced follicle development with injections of PMSG to collect oocytes at germinal vesicle (GV) stage from Graafian follicles. About 22 hours after injections. GV oocytes enclosed with several layers of cumulus cells were collected in HEPES-buffered HTF medium supplemented with FBS, 2-mercaptoethanol and dbcAMP. The collected GV oocytes were performed maturation culture in HTF medium supplemented with FBS, 2-mercaptoethanol, FSH, EGF, amino acids and vitamin for 18-20 hours. After maturation culture, oocytes were inseminated in HTF medium supplemented with BSA and resultant zygotes were cultured in KSOM medium until reached to blastocyst stage. The blastocysts were subsequently co-cultured onto the murine fetal fibroblasts as feeder cells for investigation of the implantation potencies. The ovulated oocytes, as a control, were induced by injections of hCG following PMSG and removed from ampullae of females. They were operated insemination and embryo culture likewise.

After maturation culture, over $95 \%$ of collected GV oocytes extruded their first polarbodies, thus they were considered 'matured' oocytes in the metaphase of second meiosis. There was no significant difference in fertilization rate between in vitro matured and ovulated oocytes, while the developmental rate to the blastocyst in the former were significantly lower than the latter ( $32 \%$ vs. $78 \%)$. It was noted that the embryo loss in ovulated oocytes was mainly arisen at morula stage, while that in oocytes matured in vitro was arisen at every developmental stage, especially when developed to 4-cell from 2-cell. Further, regarding post-implantation stage development. it was impossible for approximate $40 \%$ of blastocysts derived from in vitro matured oocytes to attach to the feeder cells. although almost all blastocysts from ovulated oocytes were attachable, and trophoblast cells were adhered concomitantly. It was demonstrated that maturation culture extinguished oocyte viability by degrees throughout pre-/post- implantation development. 


\section{Pro-inflammatory cytokine changes in postmenopausal women treated with hormone replacement}

therapy

Yuka Kasai ${ }^{1}$, Anna Tani ${ }^{1}$, Ayako Nakajima ${ }^{1}$, Dan Kinoshita ${ }^{1}$, Yasukazu Uemura ${ }^{1}$, Satoshi Yamamoto ${ }^{1}$, Masahiko Maegawa ${ }^{1}$. Toshiyuki Yasui ${ }^{1}$, Masaharu Kamada ${ }^{2}$, Minoru Irahara ${ }^{1}$

${ }^{1}$ The University of Tokushima; ${ }^{2}$ Namito Health Insurance Hospital

Objective: Pro-inflammatory cytokines are thought to be pathogenetic elements in osteoporosis of postmenopausal women. Monocytes, which play a key role in innate immunity by producing cytokines, are influenced by sex hormones. Since hormone replacement therapy (HRT) is widely used in postmenopausal women, we investigated the effect of HRT on the production of pro-inflammatory cytokines in postmenopausal women.

Materials and methods: Pro-inflammatory cytokines such as interleukin (IL)-1 alpha, IL-6 and tumor necrosis factor (TNF)-alpha were measured by ELISA in the supernatant of lipopolysaccharide-stimulated whole blood cells from 36 untreated and 33 HRT-treated women. Eleven of the 33 HRT-treated women were examined before and during HRT.

Results: The production of IL-1 alpha in HRT-treated women in the early $(<10$ years) and late ( $\geqq 30$ years) postmenopausal stages were significantly lower than those in untreated women at respective stages. IL-6 levels in HRT-treated women in the mid ( $\geqq 10$ and $<30$ years) and late postmenopausal stages were significantly lower than those in untreated women at respective stages. TNF-alpha levels in HRT-treated women in all postmenopausal stages were significantly lower than those in untreated women at respective stages. HRT induced a significant decrease in the production of TNF-alpha.

Conclusions: These results suggest that HRT may have an anti-inflammatory effect on pro-inflammatory cytokine production.

\section{Influence of ischemia-reperfusion treatment on ovarian functions}

Koich Nariai ${ }^{1}$, Risa Fukumoto ${ }^{1}$. Shin Onota ${ }^{1}$, Ken Watanabe ${ }^{1}$, Hiroshi Uchiyama ${ }^{2}$, Kiichi Kanayama ${ }^{2}$, Kahei Sato $^{2}$, Kiyotaka Fujise

${ }^{1}$ The Jikei University School of Medicine; ${ }^{2}$ College of Bioresource Sciences, Nihon University

Superoxide $\left(\mathrm{O}^{2} \cdot{ }^{-}\right)$and other reactive oxygen species (ROS) are important as molecules which play a role for the homeostasis including contraction of blood vessels and removal of antigens. As the ROS generated excessively in a living body, it bring about cell destruction and/or genetic mutation and causes various disease. It has been known that production of $\mathrm{O}^{2} \cdot^{-}$is an important factor in the cerebral infarction and the myocardial infarction. On the other hand, studies on a correlation between $\mathrm{O}^{2 \cdot-}$ and female infertility especially ovarian disorders are few and far between. In this study, we examined influence of $\mathrm{O}^{2} \bullet^{-}$on status of ovarian tissue and a property of ovulated ova, when treatment of ischemia-reperfusion (I/R) in the ovarian blood vessels was carried out using experimental rats. Blood flow of the left ovarian artery and vein was stemmed by a vascular clamp under laparotomy with general anesthesia using 12-15 week-old Wister rats (ischemia preparation). Four hours after the ischemia, the clamp was removed (reperfusion). There is no operation in the right ovary as the control. PMSG (50 iu) was injected subcutaneously 24 hours after this operation, and hCG (50 iu) was injected subcutaneously 48 hours after the PMSG administration. Twenty-four after the hCG injection, both of the ovaries and the oviducts were removed. Weight and histology of the ovaries, the number of ovulation points and rate of degenerating ovum were examined. Weight of the left $(\mathrm{I} / \mathrm{R})$ ovary $(38.1 \pm 9.4 \mathrm{~g})$ was significantly $(p<0.05)$ lighter than the right (control) ovary $(63.1 \pm 12.2 \mathrm{~g})$. The number of ovulation points in the left ovary $(15.0 \pm 5.0)$ was significantly $(p<0.05)$ lower than it in the right $(40.5 \pm 15.2)$. Rate of degenerating ovum in the left ampulla $(22.0 \%)$ was significantly $(p<0.05)$ higher than it in the right. Granulosa cells separated from follicular wall and a degenerating oocyte within the follicle were frequently observed in the left ovary. In conclusion, these results clarified that the $I / R$ in ovarian blood vessels caused damage to the ovarian tissue, decrease of reactivity to PMSG and hCG, and degeneration of the follicular oocyte. 
37. Reverse correlation between cycloxygenase-2 expression and degree of tumour infiltrating lymphocytes in the tumour tissue of serous ovarian adenocarcinoma

Chiho Murabayashi ${ }^{1}$. Satoshi Hayakawa ${ }^{2}$, Fumihisa Chishima ${ }^{1}$, Mika Suzuki ${ }^{1}$, Yoshiaki Ohta ${ }^{1}$, Tatsuo Yamamoto ${ }^{1}$, Norimichi Nemoto ${ }^{3}$

${ }^{1}$ Nihon University School of Medicine, Department of Obstetrics and Gynecology; ${ }^{2}$ Nihon University Advanced Medical Research Center; ${ }^{3}$ Nihon University School of Medicine, Department of Pathology

Cyclooxygenase-2(COX-2) is the rate limiting enzyme in prostanoid biosynthesis and is involved in the progression of various tumours. Prostanoids have been also reported to be involved in oxidative tissue damages. In the present study, we investigated COX-2 expression as well as 8-hydroxy-2' deoxyguanosine (8-OHDG) immunostaining and various histopathological findings in serous ovarian adenocarcinoma tissues.

Twenty four patients with primary serous adenocarcinoma of ovary were stusied under informed consents. We observed COX-2 expression in 17 of 24 samples and positive staining of 8-OHDG in all samples examined. We observed negative correlation between degree of tumour infiltrating lymphocytes and COX-2 immunostaining while no remarkable correlation in FIGO staging, histological grading, preoperative CA-125 levels or lymph nodes/ peritoneal dissemination.

As reported by others, COX-2 is an independent prognostic factor in the patients of ovarian carcinoma. From our finding of negative correlation between COX-2 expression and TIL. local ummune suppression by prostanoids may affect tumour growth. 